\title{
A Verrucous Plaque on the Lower Lip
}

\author{
Stephen K. Stacey ${ }^{\mathrm{a}, \mathrm{e}}$, Tyler A. Moss ${ }^{\mathrm{b}}$, Liesl S. Grenier ${ }^{\mathrm{c}}$, Steven D. Peine ${ }^{\mathrm{d}}$
}

\begin{abstract}
A 35-year-old male farmer with a 1-month history of an intermittently tender lower lip plaque was diagnosed with verruciform xanthoma. $\mathrm{He}$ underwent therapy with fractional $\mathrm{CO}_{2}$ laser which led to clinical resolution of the plaque followed by recurrence. Verruciform xanthoma is a rare benign entity of undetermined pathogenesis. Surgical excision is considered curative. This patient's condition may have been related to solar damage.
\end{abstract}

Keywords: Verruciform; Xanthoma; Plaque; Sun damage; Histology; Histopathology

\section{Introduction}

Verruciform xanthoma is a rare entity which typically presents as a painless solitary verrucous pink, gray, or yellow papule or plaque on the oral mucosa, especially the gingival margin. It may also occur on other locations such as the nose, ear, penis, scrotum, vulva, or extremities. The pathogenesis of verruciform xanthoma remains unclear, though it may arise due to trauma or chronic inflammation. Surgical removal is considered curative in most cases. Other destructive methods such as $\mathrm{CO}_{2}$ ablation, cryotherapy, chemical peels, and electrosurgery typically lead to recurrence. We present the case of a farmer with verruciform xanthoma appearing on the lower lip, a rare site of involvement. This area is particularly known for its association with actinic damage. This association may provide clues into the pathogenesis of verruciform xanthoma.

Manuscript accepted for publication April 16, 2015

${ }^{a} 1-503$ IN (ABN), 173D IBCT (A), USA

bRACH, Ft. Sill, OK, USA

${ }^{\mathrm{c} C R D A M C}$, Fort Hood, TX, USA

${ }^{\mathrm{d} B r o o k e}$ Army Medical Center, TX, USA

${ }^{e}$ Corresponding Author: Stephen K. Stacey, CMR 427 Box 2962, APO, AE

09630, USA. Email: stephenstacey@gmail.com

doi: http://dx.doi.org/10.14740/jmc2145w

\section{Case Report}

A 35-year-old male farmer presented with a 1-month history of an intermittently tender lower lip plaque. He denied other mucosal or cutaneous sites of involvement, and review of systems revealed no other abnormalities. Past medical history and family history were non-contributory. The lesion had been previously treated with valacyclovir, minocycline, and mupirocin for suspected impetiginized herpes simplex but there was no improvement.

Physical examination revealed a yellow verrucous and hyperkeratotic plaque which covered the entire dry mucosal lower lip without involvement of his upper lip or other oral mucosa (Fig. 1, 2). A punch biopsy specimen was obtained and submitted for histologic examination.

Histopathologic examination revealed a papillomatous lesion on mucosal skin with verrucous hyperplasia of the epidermis and elongation of the rete ridges. A superficial neutrophilic spongiosis with focal overlying parakeratosis and a dense mixed inflammatory dermal infiltrate was also observed. Foamy appearing vacuolated histiocytic cells filled the tips of the papillary dermal projections (Fig. 3, 4). No koilocytic change or atypia was appreciated. PAS, GMS, Brown-Hopps, $\mathrm{HPV}$, and spirochete stains were negative.

The patient was diagnosed with verruciform xanthoma. He underwent therapy with three sessions of fractional $\mathrm{CO}_{2}$ laser which led to near complete clinical resolution of the plaque.

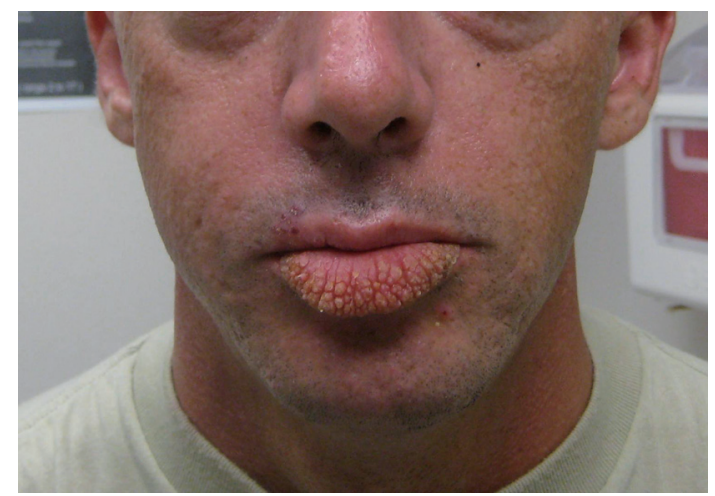

Figure 1. Yellow verrucous and hyperkeratotic plaque covering the entire dry mucosal lower lip. 


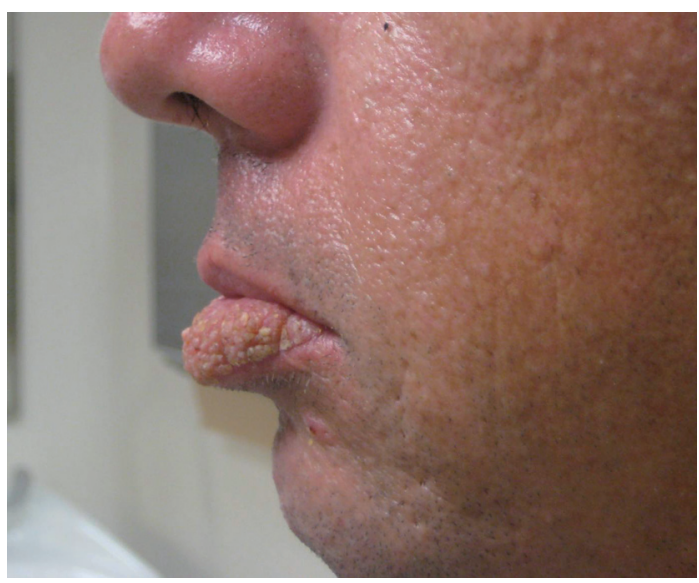

Figure 2. Profile of the patient's lesion.

However, within a few months the lesion fully recurred. The patient elected to undergo surgical treatment and is currently awaiting an excision and lip advancement procedure.

\section{Discussion}

Verruciform xanthoma is a rare entity that most commonly occurs in middle-aged adults. It typically presents as a painless solitary verrucous pink, gray, or yellow papule or plaque on the oral mucosa, especially the gingival margin [1]. Other reported locations include the nose, ear, penis, scrotum, vulva, upper and lower extremity. The lip, particularly the dry mucosal lip, is a rare site of involvement [2].

Histologically, verruciform xanthoma appears with numerous large xanthoma cells filling the papillary dermis. A mixed dermal infiltrate with lymphocytes, plasma cells, neutrophils, and eosinophils may be seen. The overlying epidermis has a verruca-like configuration consisting of hyperkeratosis, focal parakeratosis, and acanthotic epidermal invaginations. Exocytosis of neutrophils may also be seen [3].

The pathogenesis of verruciform xanthoma has not been precisely elucidated and continues to be a topic of debate.

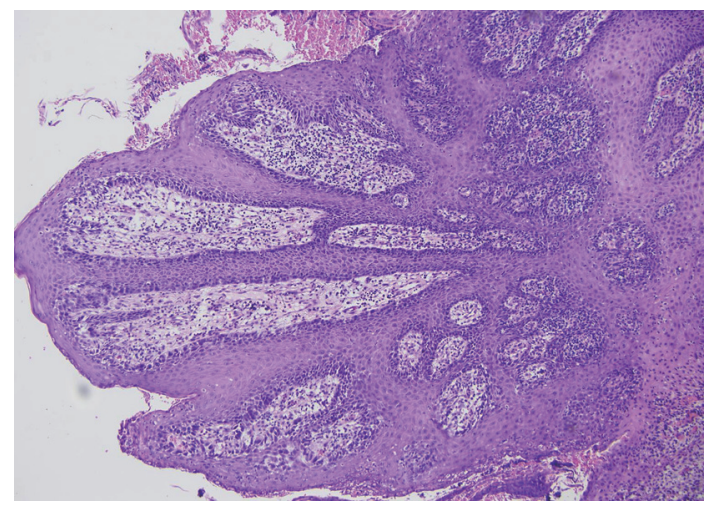

Figure 3. Histopathologic specimen of the patient's lesion revealed superficial neutrophilic spongiosis with focal overlying parakeratosis and a dense mixed inflammatory dermal infiltrate.

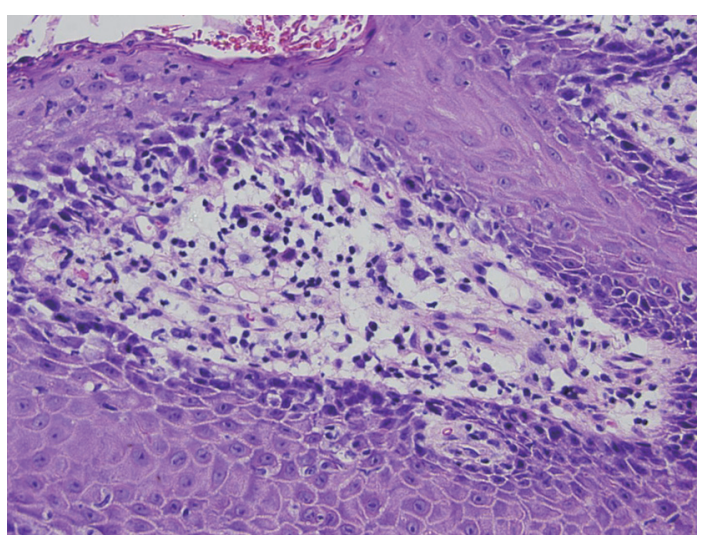

Figure 4. Histopathologic specimen of the patient's lesion. Foamy appearing vacuolated histiocytic cells fill the tips of the papillary dermal projections.

Recent reports suggest the condition to be a unique reactive process to trauma or chronic inflammation [4]. This may be supported by the occurrence of verruciform xanthoma in conditions such as discoid lupus erythematosus, lichen planus, lichen sclerosus, psoriasis, recessive dystrophic epidermolysis bullosa, graft versus host disease (GVHD) and pemphigus vulgaris. However, cases have occurred in the absence of other skin disease [5]. Verruciform xanthoma has also been associated with epidermal nevi, particularly, psoriasiform or verrucous epidermal nevi as seen in congenital hemidysplasia with ichthyosiform nevus and limb defects (CHILD) syndrome [6] and inflammatory linear verrucous epidermal nevus (ILVEN). Other proposed etiologies include human papillomavirus, bacterial colonization, lymphedema, genetic predisposition, and impaired immunity.

Recurrence is often seen with destructive methods such as $\mathrm{CO}_{2}$ ablation, cryotherapy, chemical peels, and electrosurgery. Surgical excision is considered curative for oral mucosal lesions; however, recurrence following excision of cutaneous lesions has been reported [7]. Interestingly, success has been reported with chloroxylenol surgical scrub and topical $10 \%$ povidone-iodine solution [7]. Success has also been seen with imiquimod [8].

Verruciform xanthoma is a rare entity with unknown cause. The presented case is interesting as the lesion covers an area often affected by solar damage. Previous reports have shown its presentation in areas of sun-damaged skin as well as association with actinic keratosis [9] and squamous cell carcinoma [10]. Our patient's presentation is believed to be the result of chronic solar damage due his occupation as a farmer.

\section{Conflict of Interest}

We report no conflict of interest.

\section{References}

1. Philipsen HP, Reichart PA, Takata T, Ogawa I. Verruci- 
form xanthoma--biological profile of 282 oral lesions based on a literature survey with nine new cases from Japan. Oral Oncol. 2003;39(4):325-336.

2. Colonna TM, Fair KP, Patterson JW. A persistent lower lip lesion. Verruciform xanthoma. Arch Dermatol. 2000;136(5):665-666, 669.

3. Shafer WG. Verruciform xanthoma. Oral Surg Oral Med Oral Pathol. 1971;31(6):784-789.

4. Cumberland L, Dana A, Resh B, Fitzpatrick J, Goldenberg G. Verruciform xanthoma in the setting of cutaneous trauma and chronic inflammation: report of a patient and a brief review of the literature. J Cutan Pathol. 2010;37(8):895-900.

5. Blankenship DW, Zech L, Mirzabeigi M, Venna S. Verruciform xanthoma of the upper-extremity in the absence of chronic skin disease or syndrome: a case report and review of the literature. J Cutan Pathol. 2013;40(8):745-752.
6. Fedda F, Khattab R, Ibrahim A, Hayek S, Khalifeh I. Verruciform xanthoma: a special epidermal nevus. Cutis. 2011;88(6):269-272.

7. Connolly SB, Lewis EJ, Lindholm JS, Zelickson BD, Zachary CB, Tope WD. Management of cutaneous verruciform xanthoma. J Am Acad Dermatol. 2000;42(2 Pt 2):343-347.

8. Guo Y, Dang Y, Toyohara JP, Geng S. Successful treatment of verruciform xanthoma with imiquimod. J Am Acad Dermatol. 2013;69(4):e184-186.

9. Jensen JL, Liao SY, Jeffes EW, 3rd. Verruciform xanthoma of the ear with coexisting epidermal dysplasia. Am J Dermatopathol. 1992;14(5):426-430.

10. Takiwaki H, Yokota M, Ahsan K, Yokota K, Kurokawa Y, Ogawa I. Squamous cell carcinoma associated with verruciform xanthoma of the penis. Am J Dermatopathol. $1996 ; 18(5): 551-554$ 